\title{
Series vs parallel connected organic tandem solar cells: Cell performance and impact on the design and operation of functional modules
}

\author{
Ikerne Etxebarria $^{\mathrm{a}, \mathrm{b}}$, Alice Furlan ${ }^{\mathrm{c}}$, Jon Ajuria ${ }^{\mathrm{a}, \mathrm{b}}$, Frank W. Fecher ${ }^{\mathrm{d}}$, Monika Voigt ${ }^{\mathrm{d}, \mathrm{e}}$, \\ Christoph J. Brabec ${ }^{\text {d,e }}$, Martijn M. Wienk ${ }^{c}$, Lenneke Slooff ${ }^{f}$, Sjoerd Veenstra ${ }^{f}$, \\ Jan Gilot ${ }^{\mathrm{g}}$, Roberto Pacios ${ }^{\mathrm{a}, \mathrm{b}, *}$ \\ a IK4-IKERLAN, Goiru Kalea, 20500 Arrasate-Mondragon, Spain \\ ${ }^{\mathrm{b}}$ CIC microGUNE, 20500 Arrasate-Mondragon, Spain \\ ${ }^{c}$ Molecular Materials and Nanosystems, Eindhoven University of Technology, Eindhoven 5600MB, The Netherlands \\ d Bavarian Center for Applied Energy Research (ZAE Bayern), Haberstraße 2a, 91058 Erlangen, Germany \\ e Institute of Materials for Electronics and Energy Technology (i-MEET), Department of Materials Science and Engineering, Friedrich-Alexander-Universität \\ Erlangen-Nürnberg (FAU), Martensstraße 7, 91058 Erlangen, Germany \\ ${ }^{\mathrm{f}}$ ECN, High Tech Campus 5, 5656AE Eindhoven, The Netherlands \\ ${ }^{\mathrm{g}}$ Holst Center, High Tech Campus 31, 5656AE Eindhoven, The Netherlands
}

\section{A R T I C L E I N F O}

Article history:

Received 10 April 2014

Received in revised form 21 July 2014

Accepted 31 July 2014

Available online 23 August 2014

Keywords:

Tandem organic solar cells

Two-terminal series connection

Three-terminal parallel connection

R2R module processing

\begin{abstract}
A B S T R A C T
Tandem solar cells are the best approach to maximize the light harvesting and adjust the overall absorption of the cell to the solar irradiance spectrum. Usually, the front and back subcells are connected in series in two-terminal device (2T) designs which require a current matching between both subcells in order to avoid potential losses. Alternatively, they can also be connected in parallel giving rise to a three terminal connection (3T). In principle, both designs have their assets and drawbacks in terms of device performance, design and materials' characterization. In this letter, we theoretically and experimentally confront both designs with each other (2T and 3T). Theoretical estimations show a maximum PCE of 15\% for $2 \mathrm{~T}$ and about $13 \%$ for 3T structures with ideal bandgaps for the front and back cell. However, 3T tandem devices can yield higher efficiencies than 2T for some specific material combinations whose theoretical values are between $10 \%$ and $12 \%$. Therefore, other aspects related to the fabrication feasibility are studied in order to analyze the most convenient approach for module development. The need of a conducting interlayer restricts the width of the cell and causes a $3 \%$ reduction in the geometrical fill factor of the module in comparison to the 2T approach. The R2R processing of modules with 3T cells would also require an additional laser step. Finally, a couple of existing material combinations have been experimentally implemented into $2 \mathrm{~T}$ and $3 \mathrm{~T}$ tandem devices. The limitation imposed by their specific and non-ideal bandgaps restricts the efficiency to around $7 \%$, considerably below the ideal case.
\end{abstract}

(c) 2014 Elsevier B.V. All rights reserved.

\section{Introduction}

The intense research on polymer solar cells over the past decade, not only on materials but also on device performance, has taken the efficiency towards levels that were unimaginable some years ago. In this way, power conversion efficiencies over $9 \%$ are already certified [1] and the first press releases announcing values over $10 \%$ start appearing [2]. Although these efficiencies are already appealing for energy generation purposes and integration in some other applications thanks to the advanced properties that

\footnotetext{
* Corresponding author at: IK4-IKERLAN. 20500 Arrasate-Mondragón. Spain

E-mail address: rpacios@ikerlan.es (R. Pacios).
}

organic semiconductors offer in comparison to their inorganic counterparts, researchers continue making persistent efforts in order to overcome the existing limitations. Absorption and thermalization losses are some of the most restricting issues at this moment of device development, since internal quantum efficiencies (IQE) over $90 \%$ and fill factors (FF) over $70 \%$ are routinely achieved by different labs [3]. This practically means that nearly all light that is absorbed is efficiently converted into current. Unfortunately, only the photons having energy higher than the bandgap of the photoactive material contribute to the energy conversion. Alike in inorganic semiconductors [4], tandem solar cells are the strategy followed to tackle this issue [5]. In spite of many efforts in this direction, polymer tandem cells do not yet show the impressive enhancement that would in theory be expected from the 
performance of optimized single cells. In this way, very recently reported works situate tandem record efficiencies over those obtained for single cells but still slightly over $10 \%[6,7]$. The tandem approach can therefore be an interesting option for applications with area restrictions providing that there is not a substantial increase in the price per Watt-peak produced. In most of the cases, two subcells with complementary absorption are connected in series through a recombination layer where holes from one subcell meet electrons from the other. This results in a two-terminal (2T) device for which the open-circuit voltage is the summation of each subcell, and the overall current is limited by the subcell that delivers the smallest photocurrent. In an ideal situation both subcells should deliver the same photocurrent in order to minimize losses. This photocurrent matching criterion is not easy to achieve in organic solar cells. Three-terminal (3T) tandem devices are an alternative option that eliminates the need of current matching, while potential losses in terms of unbalanced photovoltages would be less dramatic for the performance of the cell [8]. In this manuscript we compare both approaches in terms of device performance, design and materials characterization. We demonstrate, experimentally and theoretically, that the 2T design is the best option to maximize the efficiency of organic tandem devices, since they benefit from higher ideally achievable efficiencies. Theoretical maximum power conversion efficiencies (PCE) are around $15 \%$ while 3 T devices have the potential to reach $13 \%$. However, the $3 \mathrm{~T}$ design offers a larger degree of freedom in the choice of complementary absorbing materials. There are therefore some specific material combinations for which the expected efficiency for 3T devices is higher than that obtained for 2T cells. Therefore, we also analyze in detail the fabrication procedure involved for each case (2T and $3 \mathrm{~T}$ ) in order to be able to discern beforehand which design is most convenient for module development attending to the best compromise between device efficiency and level of fabrication difficulty. In addition, 3T designs also offer the possibility to independently characterize both subcells, front and back, in the same resulting structure. This would considerably ease the characterization of new materials, and would also speed up the optimization of the tandem device itself, since there is no way to check the photocurrent provided by each subcell in the $2 \mathrm{~T}$ configuration.

\section{Experimental section}

\subsection{Materials}

The following materials and solvents were used as received: P3HT (American Dye Source), PTB7 (1-material), PCDTBT (Konarka Technologies), $\mathrm{PC}_{60} \mathrm{BM}$ (SES Corporation) and $\mathrm{PC}_{70} \mathrm{BM}$ (Solenne BV), PEDOT:PSS (Al 4083 from HC Stark), chlorobenzene (Scharlau), chloroform, ortho-dichlorobenzene (Sigma-Aldrich), and diiodooctane (Sigma-Aldrich).

\subsection{Solutions}

The P3HT: $\mathrm{PC}_{60} \mathrm{BM}$ solution was prepared by mixing $15: 12 \mathrm{mg}$ in $1 \mathrm{~mL}$ of chlorobenzene. The solution was then heated overnight at $80^{\circ} \mathrm{C}$

The PTB7:PC ${ }_{60} \mathrm{BM}$ was prepared in the glove box by mixing $10: 15 \mathrm{mg}$ in $1 \mathrm{~mL}$ of solvent (97\% chlorobenzene and 3\% diiodooctane). The solution was then heated overnight at $60{ }^{\circ} \mathrm{C}$. Diiodooctane was added $1 \mathrm{~h}$ prior to the deposition of the active layer and it was left stirring at $70^{\circ} \mathrm{C}$.

The PDPP5T:PC ${ }_{60} \mathrm{BM}$ was prepared by mixing $8: 16 \mathrm{mg}$ in $1 \mathrm{~mL}$ of solvent ( $90 \%$ chloroform and 10\% ortho-dichlorobenzene).
The PCDTBT:PC ${ }_{70} \mathrm{BM}$ was prepared by mixing 7:28 $\mathrm{mg}$ in $1 \mathrm{~mL}$ of solvent (70\% ortho-dichlorobenzene and 30\% chlorobenzene).

\subsection{Devices}

ITO covered substrates were clean in subsequent acetone and isopropyl alcohol (IPA) ultrasonic baths followed by 5 min of UV ozone treatment.

\section{4. $2 T$ P3HT/PTB7 series devices}

Clean ITO substrates were covered with a thin layer of PEDOT: PSS deposited by spin-coating. This layer was dried at $120^{\circ} \mathrm{C}$ for 10 min. The P3HT:PC ${ }_{60} B M$ was deposited at different spin rates depending on the desired thickness. The P3HT:PCBM film was annealed at $140{ }^{\circ} \mathrm{C}$ for $15 \mathrm{~min}$ in a nitrogen filled glove box. For the intermediate layer fabrication; first, a $30 \mathrm{~nm}$ thick $\mathrm{ZnO}$ layer was spin cast at $2000 \mathrm{rpm}$ from a $10 \mathrm{mg} / \mathrm{ml}$ dispersion of monodisperse $5 \mathrm{~nm}$ diameter crystalline $\mathrm{ZnO}$ nanoparticles in acetone. Details on the ZnO NP synthesis are given in [9]. Secondly, a $15 \mathrm{~nm}$ thick film of $\mathrm{pH}$ neutral PEDOT was spin cast at $2000 \mathrm{rpm}$ from a 1:1 dilution of ORGACON (AGFA) and water. Next, the PTB7: $\mathrm{PC}_{70} \mathrm{BM}$ solution was spin cast at room temperature. Device fabrication was completed by thermal evaporation of $10 \mathrm{~nm}$ of $\mathrm{Ca}$ and $200 \mathrm{~nm}$ of $\mathrm{Ag}$ at room temperature under vacuum at a base pressure of $4 \times 10^{-6}$ mbar. All devices present an active area of $9.4 \mathrm{~mm}^{2}$.

\subsection{T PCDTBT/PDPP5T series devices}

On top of the dried PEDOT:PSS the PCDTBT:PC ${ }_{70} \mathrm{BM}$ was cast in nitrogen atmosphere. The resulting film was dried on a hotplate for $10 \mathrm{~min}$ at $70^{\circ} \mathrm{C}$. The intermediate layer was processed as before from a ZnO nanoparticles dispersion [10]. The ZnO layer was spun from a solution of $10 \mathrm{mg} \mathrm{mL}^{-1} \mathrm{ZnO} \mathrm{NP}$ from isopropanol on top of the dried PCDTBT active layer. The pH neutral PEDOT:PSS (Orgacon, AGFA) was diluted 1:1 with ultrapure water after which $0.2 \mathrm{~mL} \mathrm{~mL}^{-1}$ isopropanol was added to improve the wetting on the $\mathrm{ZnO}$ nanoparticles. The PDPP5T:PC ${ }_{60} \mathrm{BM}$ active layer was spun on top of that. Finally a back contact of $1 \mathrm{~nm} \mathrm{LiF}$ and $100 \mathrm{~nm} \mathrm{Al}$ was evaporated in vacuum.

\section{6. $3 T$ Р3HT/PTB7 parallel devices}

The front cell, with inverted configuration, was fabricated first. A zinc acetate $(\mathrm{ZnAc})$ solution was prepared as reported by White et al. [11]. This was spin coated onto pre-cleaned ITO substrates at $4000 \mathrm{rpm}$. The resulting film was annealed at $150{ }^{\circ} \mathrm{C}$ for $5 \mathrm{~min}$ in order to convert the $\mathrm{ZnAc}$ into $\mathrm{ZnO}$. The P3HT:PC ${ }_{60} \mathrm{BM}$ film was deposited by a doctor-blade with the substrate heated at $65^{\circ} \mathrm{C}$. Depending on the slot height between the substrate and the knife and the deposition speed, layers with different thicknesses were achieved. After depositing the photoactive layer, samples were covered with a Petri dish in order to create a solvent saturated environment. Following, PEDOT:PSS (Al 4083) with 1\% of zonyl was spin coated on top of the P3HT:PCBM layer and the samples were annealed at $140{ }^{\circ} \mathrm{C}$ for $15 \mathrm{~min}$ in the glovebox. Afterwards, $10 \mathrm{~nm}$ of Au were thermally evaporated as interlayer. In the next step, the back cell was deposited with the regular configuration. Therefore, a layer of PEDOT:PSS (Al 4083) with 1\% of zonyl was spin coated. Next, the PTB7:PC 70 BM layer was spin coated in the glovebox (at $1200 \mathrm{rpm}$ for a $100 \mathrm{~nm}$ thick layer and $1700 \mathrm{rpm}$ for $80 \mathrm{~nm}$ thick layer) followed by a drying step of $30 \mathrm{~min}$ at $60{ }^{\circ} \mathrm{C}$. Finally, the top electrode was thermally evaporated, $10 \mathrm{~nm} \mathrm{Ca/}$ $150 \mathrm{~nm} \mathrm{Ag}$. 


\subsection{Device characterization}

Current density vs voltage curves (JV) were measured under simulated solar light from a Xe lamp under AM1.5G illumination using a Keithley 2420 Source-Measure Unit. The calibration of the light intensity was carried out with an NREL certified monocrystalline silicon photodiode. No mismatch correction was done. For the single junction cells the accurate short-circuit current density $\left(J_{\mathrm{sc}}\right)$ was determined from the External Quantum Efficiency (EQE) by convolution with the AM1.5G solar spectrum. The EQE was measured using a Xe lamp illuminating at $30 \mathrm{~Hz}$ and an Oriel Cornerstone $260 \frac{1}{4}$ monochromator. Signal was detected using a Merlin lock-in amplifier and processed with Oriel software. For 2T series devices, the simulated solar light spectrum was tuned to provide appropriate illumination to each subcell. This was achieved by adjusting the voltage over the lamp in such a way that both the wide bandgap and the narrow bandgap single junction reference cell gave the exact $J_{\mathrm{sc}}$ as determined from the EQE measurement. The $J V$ curves of the tandem solar cells were measured under illumination through a mask of identical dimensions to the device area determined by the overlap of the ITO and the Al electrodes, to avoid extra current generation due to the high lateral conductivity of the pH neutral PEDOT and the PEDOT/Au/ PEDOT interlayer.

\section{Parallel connection: $3 T$ devices}

The 3T structure is shown in Fig. 1. Two subcells (front and back) are connected in parallel through a transparent conducting interlayer that acts as a common anode to the two subcells. The front cell can be independently characterized by contacting at the front cell cathode and the common anode. In similar terms, the back cell can be individually characterized by contacting the cathode back cell and the common anode. Finally, if both cathodes (front and back cells) are short circuited to make the negative electrode, the tandem cell can be measured by accessing to the latter and to the common anode. Apart from being able to characterize both subcells independently, this structure allows the extraction of the sum of the $J_{\text {sc }}$ belonging to both subcells, if the open-circuit voltage, $V_{\mathrm{oc}}$, is not very different. It is important to note that even if the $V_{\mathrm{oc}}$ of the two subcells does not match, power can still be drawn from the two subcells without satisfying the photocurrent matching and without excessive overall losses. The most challenging task to accomplish such a structure is to build an effective transparent and conducting layer as a common electrode. Optically, as in 2T, it must allow light to be transmitted. Electrically, in contrast to $2 \mathrm{~T}$ where no lateral conductivity is needed, it should act as an efficient collecting contact for charge carriers from both subcells, while being able to withstand the solution processing of the second subcell and protect the one underneath. In one of the previous works published with this design [8], Sista and co-workers did not use any buffer layer at the front cell to improve electron charge injection/collection at this electrode. Moreover, they used a vacuum deposited buffer layer of $\mathrm{V}_{2} \mathrm{O}_{5}$ as a hole collecting layer for the back cell at the intermediate/ common electrode. In contrast, we use a thin $\mathrm{ZnO}$ layer on top of the transparent ITO electrode to improve electron injection/ collection at the front cell. Furthermore, our intermediate electrode comprises 3 very thin layers of PEDOT:PSS, evaporated Au, and PEDOT:PSS. With a careful adjustment of their thickness to 40 , 10 and $40 \mathrm{~nm}$ respectively, we demonstrate sufficient electrical conductivity and transparency of the interlayer and successful protection for the front subcell against the solution processing of the back subcell above it. In addition, the photoactive material of the back cell and hence the absorption profile of the whole stack is considerably different.

\section{Series vs parallel connection}

According to Dennler et al. [12], we simulated the achievable PCE for 2T and 3T tandem devices as a function of the bandgap of the donor materials used for the front and the back cells. However, we made three extensions/specifications to Dennler's model: (i) The highest open circuit voltage of our subcells 1 and $2\left(V_{\mathrm{oc}, 1 /}\right.$ 2) is assumed to be reach for a solar cell with an idealized donoracceptor couple. It can be expressed by the subcells donor bandgap $\left(E_{\text {gap }, 1 / 2}\right)$ as experimentally shown by Veldman et al. [13]. Therefore, we used for the subcells open-circuit voltages: $V_{\text {oc, } 1 / 2}=\left(E_{\text {gap }, 1 / 2}-0.6 \mathrm{eV}\right) / \mathrm{e}$ with e being the elementary charge. (ii) The parameters $\mathrm{EQE}=65 \%, \mathrm{IQE}=85 \%$ and $\mathrm{FF}=65 \%$ were fixed for both subcells. Those values represent the highest used values by Dennler et al. and are in good in agreement with standard organic solar cells. (iii) Since Dennler et al. only simulated series connected tandem structures, the model was extended for parallel connected devices by the equations $V_{\mathrm{oc}, \tan , 3 \mathrm{~T}}=\min \left[V_{\mathrm{oc}, 1} ; V_{\mathrm{oc}, 2}\right]$ and $J_{\mathrm{sc}}$, $\tan , 3 \mathrm{~T}=J_{\mathrm{sc}, 1}+J_{\mathrm{sc}, 2}$.

Fig. 2 shows the PCE as a function of the bandgap of the front ( $y$-axis) and the back cell ( $x$-axis) for both designs, series (left) and parallel (right). Even if these simulations represent the upper limit with modest assumptions, very meaningful conclusions can be extracted. Firstly, the highest achievable efficiencies are next to the diagonal for the $2 \mathrm{~T}$ design. This means that different absorbing materials with different bandgaps are needed for high efficient 2T tandem devices. More importantly, the maximum achievable efficiency is around $15 \%$ for a combination of materials strictly restricted to the darkest red area in Fig. $2\left(1.4 \mathrm{eV}<E_{\mathrm{g} 1}<1.7 \mathrm{eV}\right.$; $1.1 \mathrm{eV}<E_{\mathrm{g} 2}<1.5 \mathrm{eV}$ ). The optimum bandgap of the front cell should be between 1.4 and $1.7 \mathrm{eV}$, while the bandgap of the back cell should be between 1.1 and $1.5 \mathrm{eV}$ (best for $E_{\mathrm{g} 1}=1.66 \mathrm{eV}$; $E_{\mathrm{g} 2}=1.33 \mathrm{eV}$ ). If we allow a $15 \%$ variation (still $13 \%$ efficient tandem devices) the choice of materials is still rather limited (orange, third color from the top).

In contrast, for the parallel connection and a given bandgap, its most efficient complementary material is always on the diagonal.
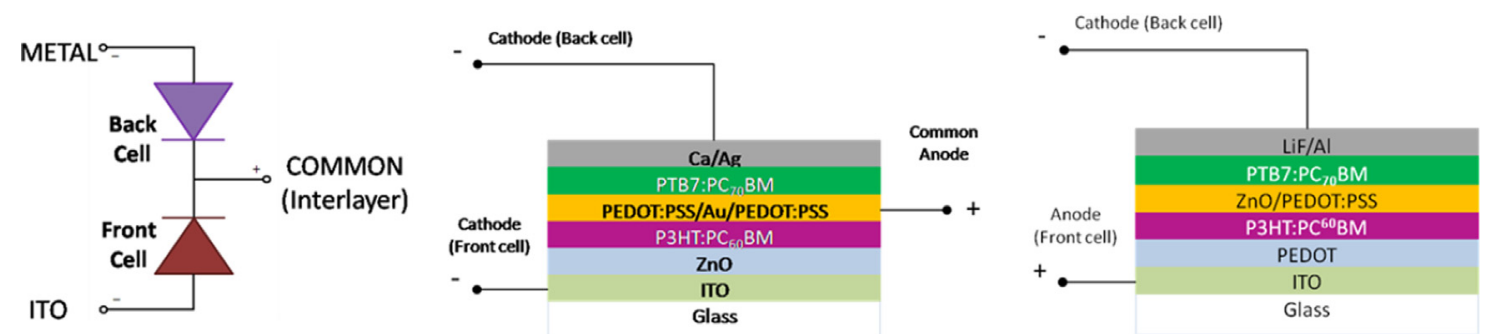

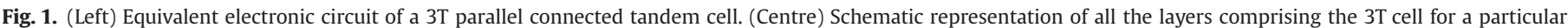
material combination. (Right) Schematic representation of all the layers comprising the $2 \mathrm{~T}$ cell for a particular material combination. 

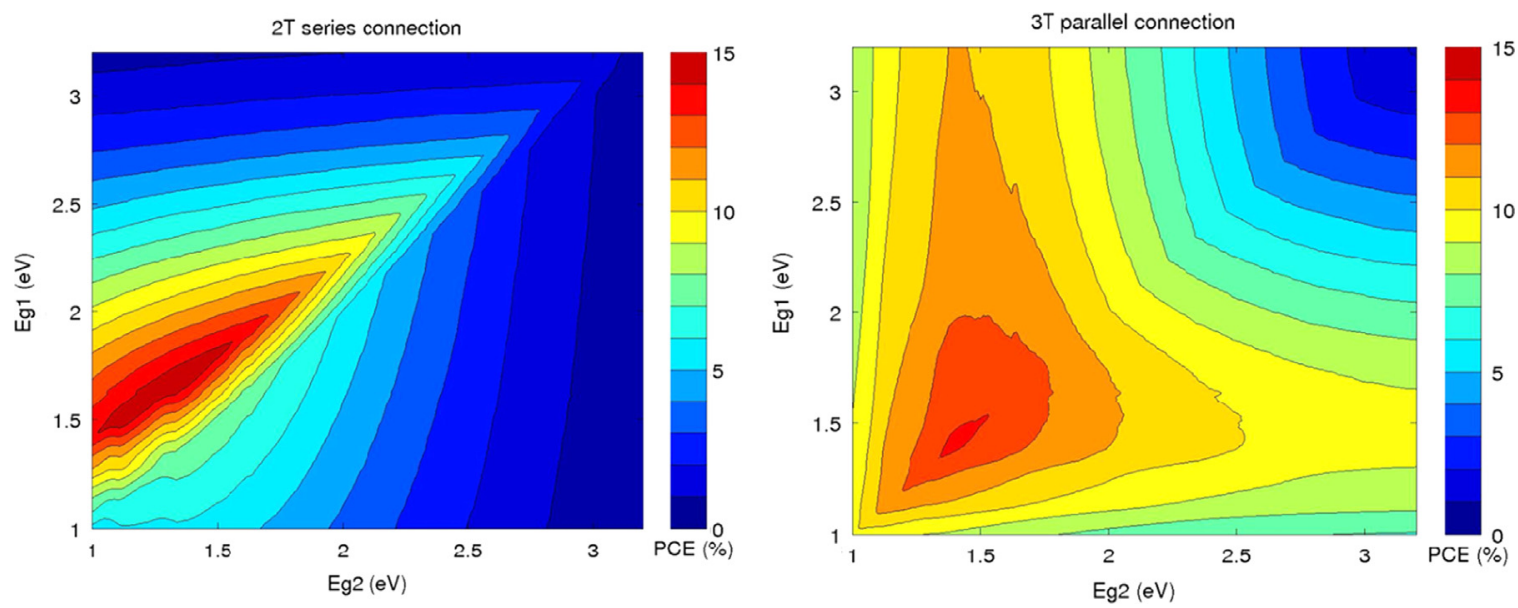

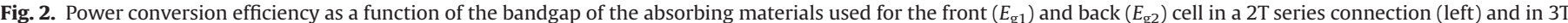
parallel connection (right) cell. (For interpretation of the references to color in this figure legend, the reader is referred to the web version of this article.)

Therefore, very similar materials have to be stacked in parallel connection if the full potential of tandems shall be exploited. The highest achievable efficiency is $13.2 \%$, but the choice of materials is larger than that in the series connection for similar efficiencies. The optimum bandgap of the front and back cell would be between 1.2 and $1.6 \mathrm{eV}$ (best for $E_{\mathrm{g} 1}=E_{\mathrm{g} 2}=1.41 \mathrm{eV}$ ). If we allow a similar variation of $15 \%$, the number of possible combinations covers most of the materials synthesized nowadays (light orange, fourth color from the top). Thus, the possible material combinations in order to achieve high efficiencies over 13\% is larger for $2 \mathrm{~T}$ that for 3T, whereas for moderate efficiencies around $11 \%$ the materials operating window is larger for 3T than for 2T. Moreover, for some material combinations, the expected PCE is higher for 3T that for $2 \mathrm{~T}$. As a representative example, let us consider a typical blue absorber of $E_{\mathrm{g} 1}=2.4 \mathrm{eV}(500 \mathrm{~nm})$ and a red absorber of $E_{\mathrm{g} 2}=1.5 \mathrm{eV}(800 \mathrm{~nm})$. The PCE expected for $2 \mathrm{~T}$ devices would be $8 \%$, while the $3 \mathrm{~T}$ cells would yield $11 \%$. Thus, the $3 \mathrm{~T}$ approach is still an interesting option attending to strictly efficiency criteria that cannot be ruled out. Even though the bandgap of organic semiconductors can be chemically tuned at wish up to certain extend during the synthesis, it can be difficult to be as precise as to obtain the ideal values commented above. In this case, when device engineers have to cope with bandgap deviations, the 3T design can be useful to extract the maximum power from a given combination of materials.

The $2 \%$ difference in maximum PCE between the two device types is caused by the constraint of matching $J_{\mathrm{sc}}$ and matching $V_{\mathrm{oc}}$ for 2T and 3T devices for their highest efficiencies, respectively. For 3T devices this is fulfilled if both subcells have the same bandgap. However, even if they have the same absorption spectra, the back cell receives fewer photons. Thus, the back cell acts just as a boost of the front cell by enhancing a small amount of the 3T-tandem's $J_{\text {sc }}$. The same result would be reached by having a single cell with higher EQE. For the 2T, in contrast, the bandgaps have to be different in order to have the same $J_{\mathrm{sc}}$ for both subcells. Thus, their absorption spectra can be complementary and the sun spectrum can be exploited more efficiently. The conversion loss in $V_{\text {oc }}$ by at least $0.6 \mathrm{~V}$ to the corresponding bandgap even increases the relative difference between the PCE maxima of $2 \mathrm{~T}$ and $3 \mathrm{~T}$, as the $J_{\text {sc }}$ of the 3T tandem cell is in general larger for 2T devices.

Practically, the 3T with identical absorbers is an interesting approach if the front cell cannot absorb all the light, i.e., if very thin active films are required for the front cell for example as a consequence of limited transport properties of the active material. Then, the back cell acts as a back-up absorber and assists in harvesting all the light, yielding a current gain for the same voltage. A difference in $V_{\text {oc }}$ does not penalize in excess the tandem performance, and therefore the maxima are located on the diagonal. In the case that the front cell absorbs everything, the back cell is redundant and does not contribute to the device performance. In a $2 \mathrm{~T}$ device with complementary absorbers, although the front cell absorbs everything, the back cell still contributes to the performance by absorbing part of the subbandgap photons transmitted by the front cell and thereby increases the $V_{\text {oc. }}$ In this situation, $2 T$ devices also make a more efficient use of the energy of absorbed photons. It is therefore important to split the overall absorption of the cell into the spectra of both active materials and balance the photocurrent produced in each subcell in order to take full advantage of this design and achieve maximum efficiencies.

In brief, 2T devices offer the potential to achieve higher maximum efficiencies than 3T when ideal absorbers are used, but thanks to the larger operational window for material choice, the parallel connection in tandem solar cells can be more efficient than the series connection for certain material combinations. In this way, other considerations as fabrication feasibility have to be taken into account before ruling one of them out. Before facing these issues, let us consider two particular examples.

\section{P3HT-PTB7}

In many cases, most of the materials yielding the highest efficiencies are produced from chemical research groups in very small amounts which considerably limit their access and reproducibility. The specific material combination P3HT-PTB7 was chosen because they both are easily commercially available and the beforehand mentioned issues are circumvented. The bandgap of P3HT is $E_{\mathrm{g} 1}=1.9 \mathrm{eV}$, and even if higher record efficiencies are reported [14], standard efficiencies for P3HT:PCBM devices are between $3.5 \%$ and $4.5 \%$. Our $3 \mathrm{~T}$ design demands the inversion of the front cell, swapping the polarity in comparison to cells with regular configurations and making use of the inverted approach. These cells design gives very similar or slightly higher efficiencies [15].

PTB7 is benzodithiophene derivative from which devices up to 9.2\% efficient are successfully processed [1]. Its optical bandgap lies at $E_{\mathrm{g} 2}=1.6 \mathrm{eV}$ [3]. Best efficiencies reported for the configuration we use in our tandem design with electrodes of PEDOT:PSS and $\mathrm{Ca} / \mathrm{Ag}$ has been shown to be $7.4 \%$. Most likely due to differences in the molecular weight and impurities of the batches 

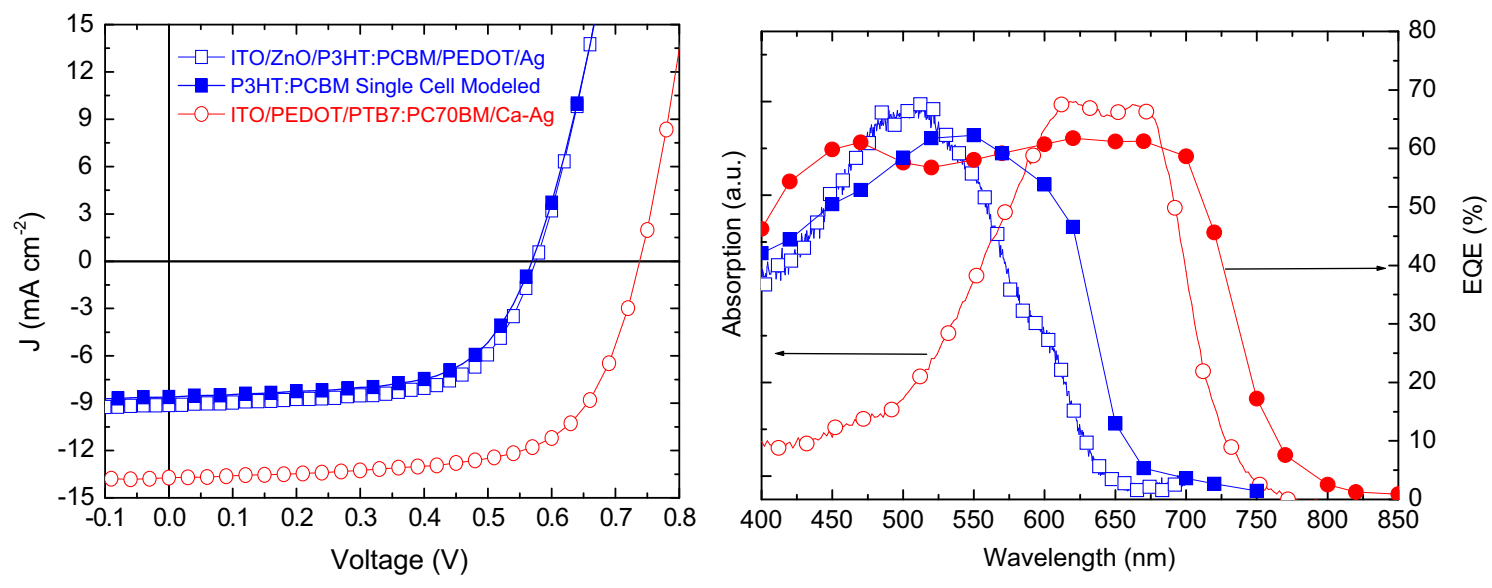

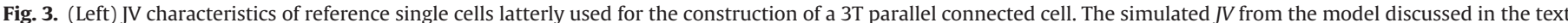

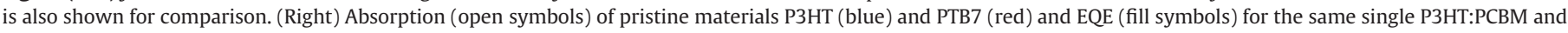
PTB7:PC70BM reference cells. (For interpretation of the references to color in this figure legend, the reader is referred to the web version of this article.)

Table 1

Figure of merits for the reference single cells shown in Fig. 3 (average of 10 diodes).

\begin{tabular}{llccl}
\hline Device & $\boldsymbol{V}_{\text {oc }}[\mathbf{V}]$ & $\boldsymbol{J}_{\text {sc }}\left[\mathbf{m A ~ \mathbf { ~ c m } ^ { - 2 } ]}\right.$ & FF & PCE [\%] \\
\hline ITO/ZnO/P3HT:PCBM/PEDOT/Ag & 0.57 & 9.12 & 0.63 & 3.3 \\
ITO/PEDOT/PTB7:PC70BM/Ca/Ag & 0.74 & 13.71 & 0.66 & 6.7 \\
\hline
\end{tabular}

we commercially acquire, our standard efficiencies for this material are around $6.7 \%$.

Fig. 3 shows the $J V$ characteristics of reference single cells of P3HT:PCBM and PTB7:PC70BM with the same electrodes as those that will be later implemented into the $3 \mathrm{~T}$ configuration, along with the EQE and the absorption spectra of both materials. The photovoltaic performance is summarized in Table 1 .

Before attempting the fabrication of tandem cells with varying thickness of the photoactive films for the front and back cell, we combined an electrical and optical model in order to be able to predict theoretically the best layer thicknesses for the highest performance in a tandem cell [16].

The refractive index, and the extinction coefficient were used together with the IQE and the thickness dependence of $J V$ curves for optimized single junction devices of varying thickness. For each thickness, the spectrally averaged IQE was determined as the ratio between the experimental short circuit current, determined by convoluting the solar spectrum with the EQE of the solar cell, and the absorbed photon flux, which was calculated via optical modeling of the entire layer stack using the wavelength dependent refractive index and extinction coefficient. The modeling therefore accounts for the electrical and optical interactions between the individual subcells in the tandem configuration and calculates the $J V$ curve of the tandem solar cell as function of the layer thickness of both subcells. The calculations assume smooth interfaces between all layers and an intermediate contact that is free of resistive losses. Finally, the model was enabled to reconstruct the $J V$ curve of the tandem when either the subcells were connected in series as well as in parallel, taking into account the restrictions of each operating mode already mentioned in the previous section.

Fig. 4 shows a summary of the obtained results. Firstly, it has to be pointed out that the previous model used to calculate the maximum achievable efficiency only as a function of the bandgap of both active films, predicted a PCE for this combination of materials of around $12 \%$ for the series connection and $11 \%$ for the parallel connection. These numbers are well above the efficiencies reported for single cells, thus the effort to attempt tandem is justified. However, the combination of the electrical and optical model predicts a maximum efficiency of $6.4 \%$ for the series connection and $5.8 \%$ for the parallel connection, providing the optimum thickness for each case are used. The latter refers to a more realistic situation, since the former assumes a FF and a constant EQE of $65 \%$ that as we have seen, is not always strictly achievable in single experimental devices, and even more difficult when they are implemented in tandem. The processing restrictions to use suboptimal contacts frequently leads to losses in FF and $V_{\text {oc }}$ with respect to optimized single devices with ideal contacts. Furthermore, the former also assumes total transmission at the interlayer, which can be plausible for the series connection but not feasible for the parallel configuration. The transmission of only $10 \mathrm{~nm}$ of gold used in the interlayer in the visible part of the spectrum is around $70 \%$. All in all, it can be considered as an upper limit for the performance of tandem devices.

Interestingly, the parallel connection is less sensitive to the thickness of the front cell. Maximum efficiencies are obtained for a range of P3HT:PCBM thicknesses from 100 to $200 \mathrm{~nm}$, while for the series connection this allowance is restricted to $90-130 \mathrm{~nm}$. In contrast, variations in the thickness of the PTB7:PC70BM back cell are less significant for the series connection. For a given thickness of $110 \mathrm{~nm}$ for the P3HT:PCBM cell, we are limited to a film thickness of PTB7:PC70BM of 70-90 nm, but we could use between 90 and $150 \mathrm{~nm}$ for the series connection.

In order to test this experimentally, series and parallel tandem cells were made using the experimental details given in Section 2. Different layer thicknesses were used for the front and back cell.

Fig. 5 shows the best experimental results achieved for active film thicknesses of 100 and $80 \mathrm{~nm}$ for the front and the back cell respectively in $3 \mathrm{~T}$ parallel configuration. Theoretical results obtained from the modeling are also shown for comparison. The $J V$ curve for the experimental front cell agrees rather well with the model, as we can also see for the reference cell in Fig. 3 left. However, for the back cell, there is a small variation in the FF that also affects the $J_{\mathrm{sc}}$ and $V_{\mathrm{oc}}$, and hence the PCE. This deviation most likely arises from resistive losses at the intermediate layer that are not taken into account in the model. The effect is less pronounced in the front cell since it delivers a smaller current. In real, the conductivity of this very thin layer of $\mathrm{Au}(10 \mathrm{~nm})$ is not sufficient as to efficiently extract the current photogenerated at both cells. This generates losses in $J_{\mathrm{sc}}$ and also $V_{\mathrm{oc}}$ since there might be some charge accumulation at the interface. We must be very close to the maximum current that the intermediate contact can extract since thicker active films, that according to the model should produce 

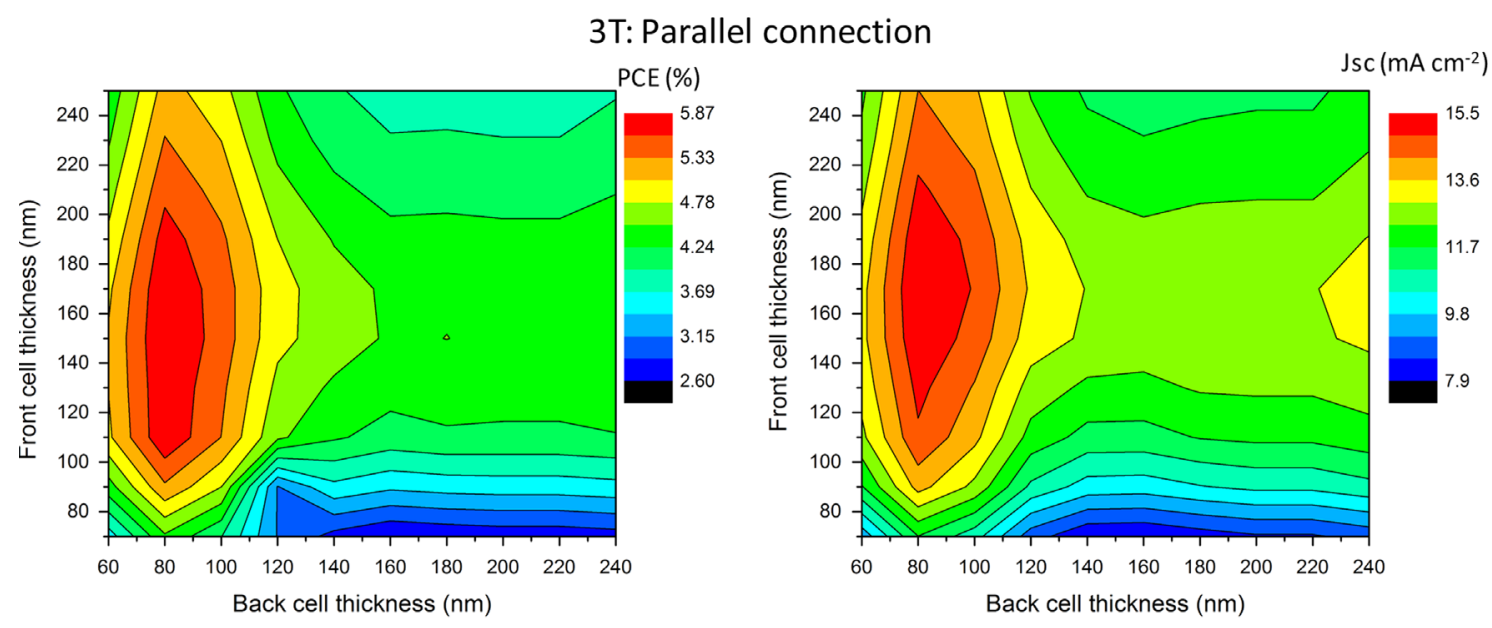

\section{T: Series connection}
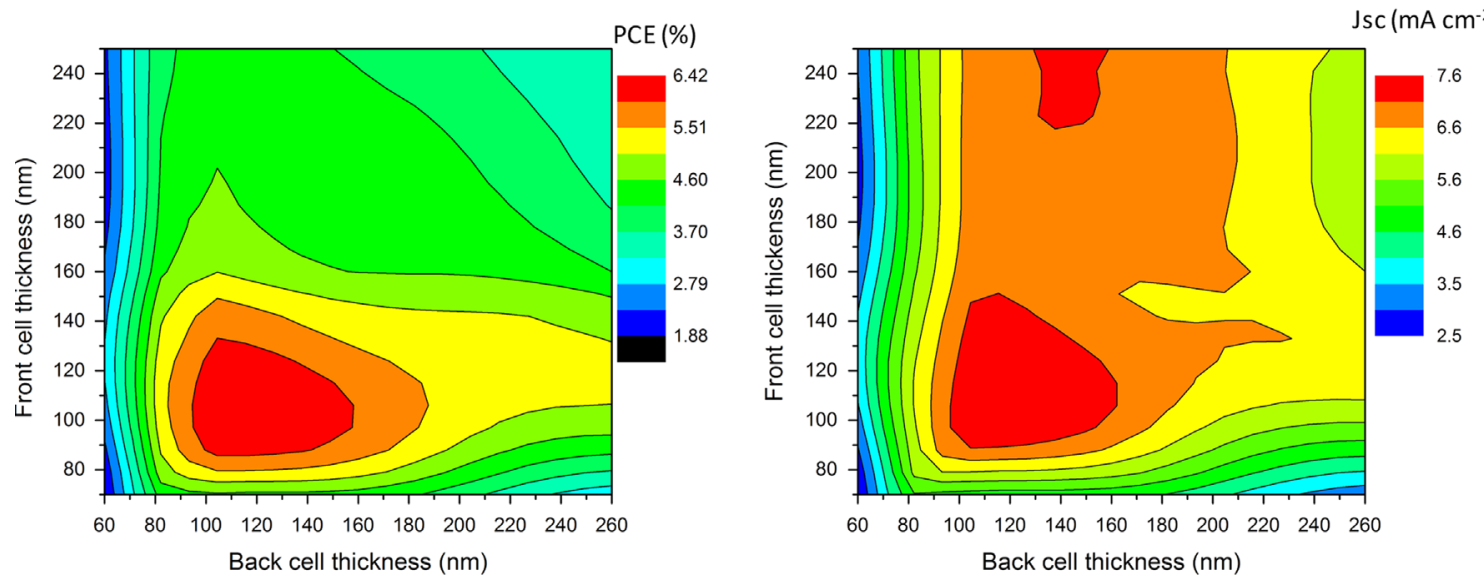

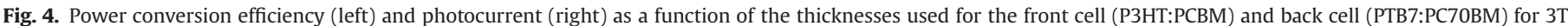
parallel connection (top) and 2T series connection (bottom).

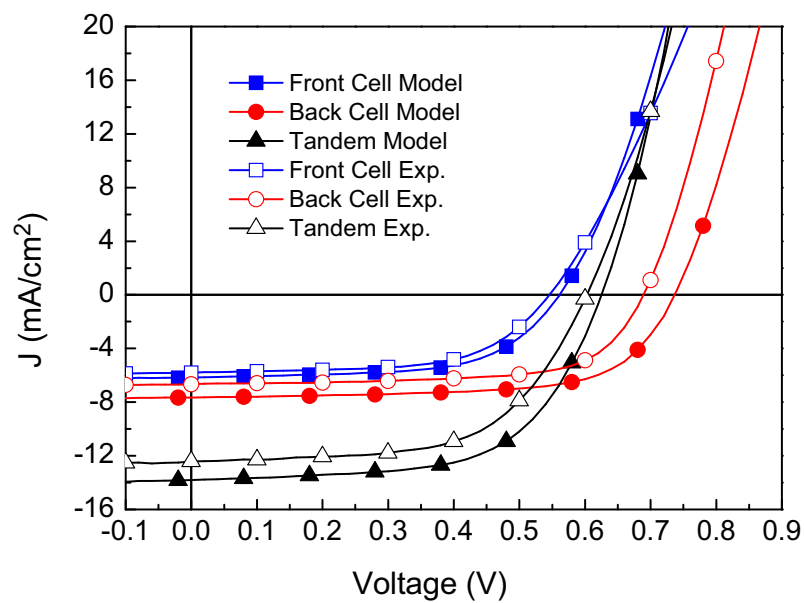

Fig. 5. Experimentally measured and simulated $J V$ characteristics for a $3 T$ parallel connected tandem cell comprised by a front cell of P3HT:PCBM (100 nm) and a back cell of PTB7:PC70BM (80 nm).

larger photocurrents (Table 2), yield indeed very similar experimental results. This is the reason why even though the model theoretically predicts more efficient tandem devices for a different combination of thicknesses, we find the best compromise for a slightly different one. The loss of performance in the back cell due to resistive issues at the interlayer is consequently reflected in the
Table 2

PCE and $J_{\text {sc }}$ for experimentally measured and simulated P3HT:PCBM and PTB7: PC70BM 3T parallel connected cells for different thicknesses of the active layers in the front and the back cell.

\begin{tabular}{lllll}
\hline Thickness $[\mathbf{n m}]$ & $\boldsymbol{J}_{\mathbf{s c}}\left[\mathbf{m A ~ \mathbf { ~ c m } ^ { - \mathbf { 2 } } ]}\right.$ & & PCE [\%] & \\
\hline Front/back & Measured & Model & Measured & Model \\
$\mathbf{1 6 0} / \mathbf{8 0}$ & 12.01 & 15.4 & 4.17 & 5.8 \\
$\mathbf{1 0 0} / \mathbf{8 0}$ & 12.43 & 14 & 4.48 & 5.5 \\
$\mathbf{1 2 0} / \mathbf{1 0 0}$ & 11.67 & 14 & 4.31 & 5.4 \\
\hline
\end{tabular}

tandem configuration, and maximum efficiencies calculated from the model are not experimentally achieved.

Fig. 6 shows the results for 2T tandem cells connected in series (right), as well as the performance of individual representative subcells that will be later implemented as front and back cells in tandem configuration (left). A regular optimized PTB7:PC70BM cell is also shown as a reference. Experimental and theoretical results for a $140 \mathrm{~nm}$ thick device of P3HT:PCBM and a $110 \mathrm{~nm}$ thick of PTB7:PC70BM are in good agreement. It has to be pointed out that in order to be able to compare the modeling of the back cell with the experimental results, since the $2 \mathrm{~T}$ series connection does not allow measuring the back cell in the tandem, the effect of light absorption at the front cell was accomplished by depositing an identical active film of P3HT:PCBM at the other side of the substrate. As in the case of 3T, the best experimental results are achieved for film thicknesses different to those used in the simulation. While the latter predicts maximum efficiencies of 

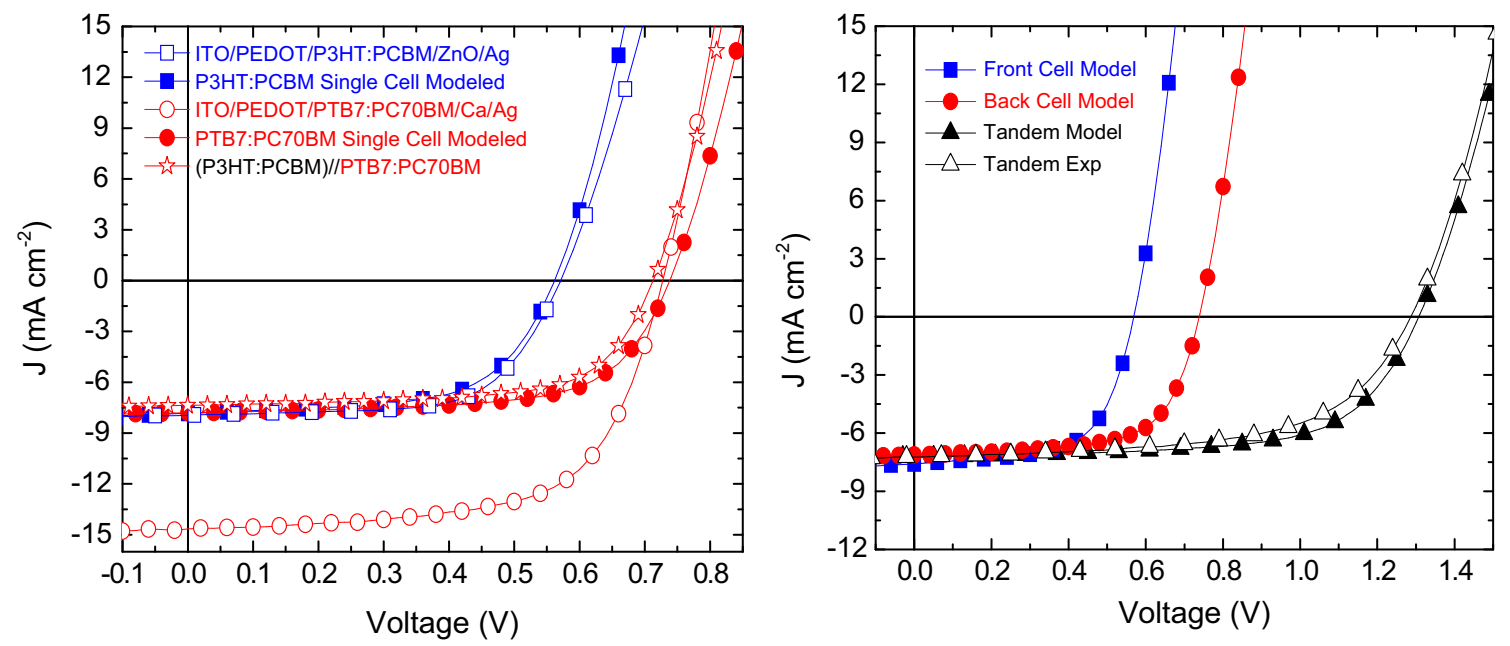

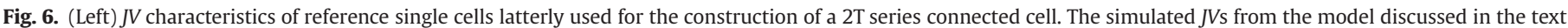
are also shown for comparison. (Right) JV characteristics for a 2T series connected cell comprised by a front cell of P3HT:PCBM and a back cell of PTB7:PC70BM.

Table 3

Photovoltaic performance of modeled and experimentally made $2 \mathrm{~T}$ tandem cells.

\begin{tabular}{llllll}
\hline Device (thickness active films) & {$[\mathbf{n m}]$} & $\boldsymbol{V}_{\text {oc }}[\mathbf{V}]$ & $\boldsymbol{J}_{\mathbf{s c}}[\mathbf{m A ~ c m}$ & $\mathbf{F F}$ & PCE [\%] \\
\hline 2T series simulated (90/110) & 1.30 & 7.53 & 0.64 & 6.4 \\
2T series experimental (90/110) & 1.27 & 6.66 & 0.59 & 5.0 \\
2T series simulated (140/110) & 1.30 & 7.20 & 0.60 & 5.7 \\
2T series experimental (140/110) & 1.29 & 7.22 & 0.58 & 5.4 \\
\hline
\end{tabular}

$6.4 \%, J_{\mathrm{sc}}$ of $7.53 \mathrm{mAcm}^{-2}, V_{\mathrm{oc}}$ of $1.3 \mathrm{~V}$ and $\mathrm{FF}$ of 0.64 for a combination of thicknesses of 90 and $110 \mathrm{~nm}$, our best experimental results are achieved for 140 and $110 \mathrm{~nm}$ respectively. For this case, the simulation yields FF of 0.60 and PCE of $5.7 \%$. Experimentally, we measured PCE of $5.4 \%$, FF of $0.58, V_{\text {oc }}$ of $1.29 \mathrm{~V}$ and $J_{\mathrm{sc}}$ of $7.2 \mathrm{~mA} \mathrm{~cm}^{-2}$. Both simulated and experimental are compared in Fig. 6 right. Results are shown in Table 3. For other different thicknesses of active films for front and back cells, our experimental results deviate further from the simulations, specially for thinner films of the front cell. The largest differences are observed in $V_{\text {oc }}$ and FF. This is clearly assigned to the effect of the interlayer and the sensitivity of it to small changes in the $\mathrm{ZnO}$ layer. This layer is very difficult to process and reproduce. It is clear that as in the previous case, the model assumes interlayers with no resistive losses, but we are suffering from a non-optimized $\mathrm{ZnO}$ layer in terms of doping and non-allignment of energy levels that causes an important charge loss at this layer.

\section{PCDTBT-PDPP5T}

Next, a benchmark material synthesized at the Eindhoven University of Technology (PDPP5T) was used with PCDTBT to compare the performance of a highly efficient system. This combination has reported efficiencies over $7 \%$ in $2 \mathrm{~T}$ series connection [17]. PDPP5T is a small bandgap diketopyrrolopyrroleoligothiophene copolymer that provides single junction solar cells over $5.3 \%$. Its bandgap lies at $E_{\mathrm{g} 2}=1.46 \mathrm{eV}$. PCDTBT is a high bandgap carbazole-benthothiadiazole copolymer with $E_{\mathrm{g} 1}=1.9 \mathrm{eV}$ that also has the potential to achieve efficiencies over $7 \%$ in single cells $[18,19]$, however, standard devices are in the range of $5.5 \%$ [17]. According to the initial model exclusively based on the bandgap of both materials, this combination should be one of the quite well performing possible choices with maximum achievable efficiencies of $13 \%$ for the $2 \mathrm{~T}$ series connections and around $12 \%$ for the $3 \mathrm{~T}$ parallel connection (Fig. 2). As in the previous case, these numbers are also considerably larger than those reported for single cells. Thus, the tandem design is the only remaining approach to maximize the power that one can extract from these materials. However, constant EQE of $65 \%$ over the visible and FF of 0.65 are also difficult to obtain in single devices. When we implemented data from real devices into tandem simulations, maximum feasible efficiencies are around $6.8 \%$ for optimum thickness of 140 and $90 \mathrm{~nm}$ for the front and back cell respectively in $2 \mathrm{~T}$ series connection, and $6.3 \%$ for the $3 \mathrm{~T}$ parallel connection with film thicknesses of over $150 \mathrm{~nm}$ for the front cell and $100 \mathrm{~nm}$ for the back cell (Fig. 7). These numbers are still above those reported for standard single cells made of each of these materials. Fig. 8 shows the experimental and simulated $J V$ characteristics for series (left) and parallel (right) connections using the optimum active layer thickness. For this set of devices we do not suffer from significant losses at the interlayer since experimental and simulated results agrees rather well (Table 4).

In summary, the $2 \mathrm{~T}$ series connection outperforms in terms of power conversion efficiencies the 3T parallel connection for the 2 set of materials tested. If a proper material combination with adequate complementary bandgaps is used, the tandem connection also outperforms the performance of single devices. However, the 3T design cannot still be completely disregarded for module fabrication since it offers more flexibility for the choice of the back cell and higher potential efficiencies for some material combinations. Although processing issues currently limit our experimentally achieved efficiency in comparison to the theoretically predicted one, the effect at module level must be studied. Existing limitations can be eventually solved and feasibility issues involved in the fabrication procedure can be the critical points to optimize the price per Watt-peak.

\section{Effect of parallel connection at module level}

In order to figure out the restrictions that an intermediate conducting electrode impose in the design and fabrication of operating modules, we have studied in detail the geometrical fill factor determined by the intermediate electrode and the impact in a potential R2R module fabrication. In large scale manufacturing, slow and batch-type processes like evaporation and spin coating should be prevented. We therefore consider a production process in the following way: substrate, screen printed silver, slot die coated PEDOT, $\mathrm{ZnO}$, photoactive layer, PEDOT, $\mathrm{ZnO}$, photoactive layer and PEDOT, and screen printed silver for a $2 \mathrm{~T}$ configuration. 


\section{T: Parallel connection}
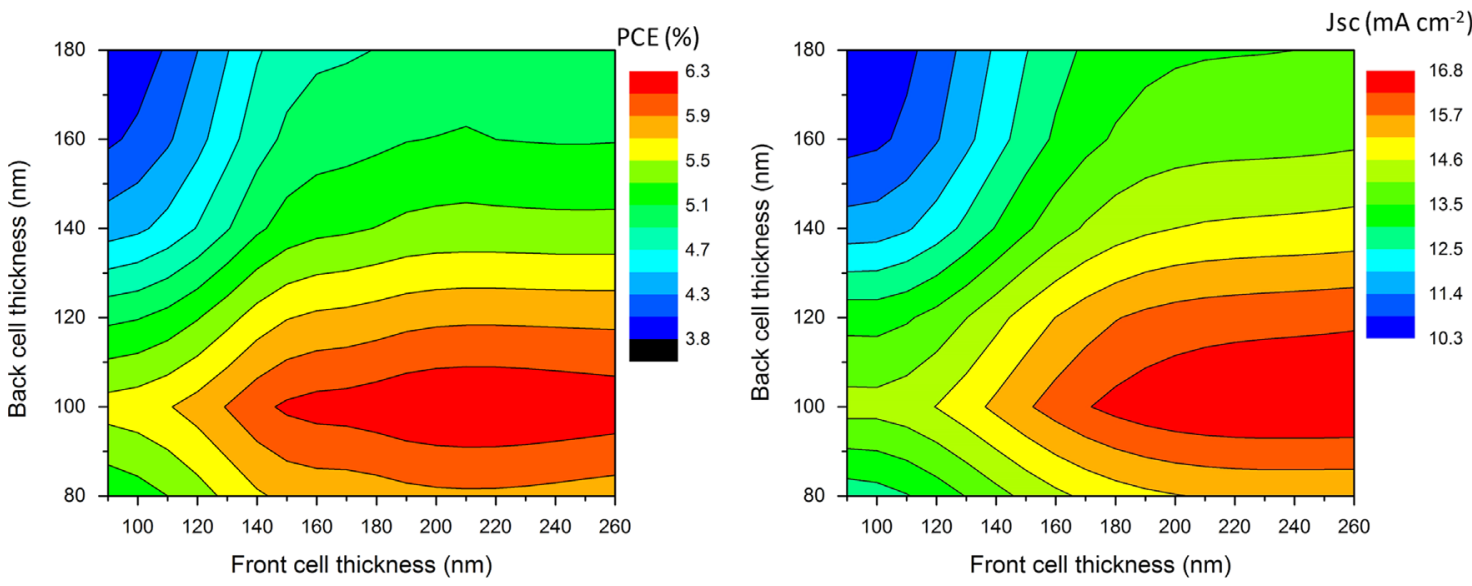

\section{T: Series connection}
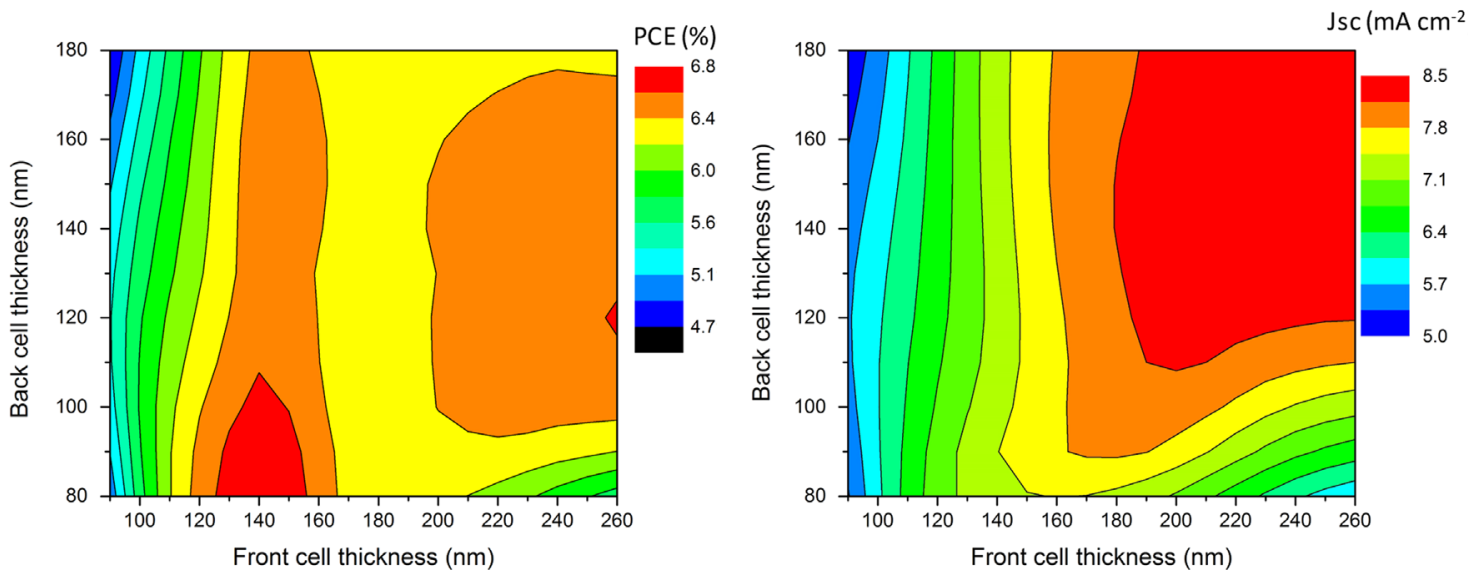

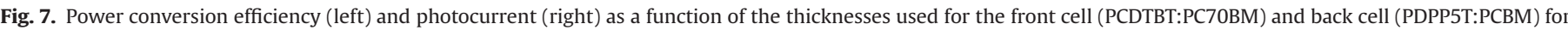
3T parallel connection (top) and 2T series connection (bottom).
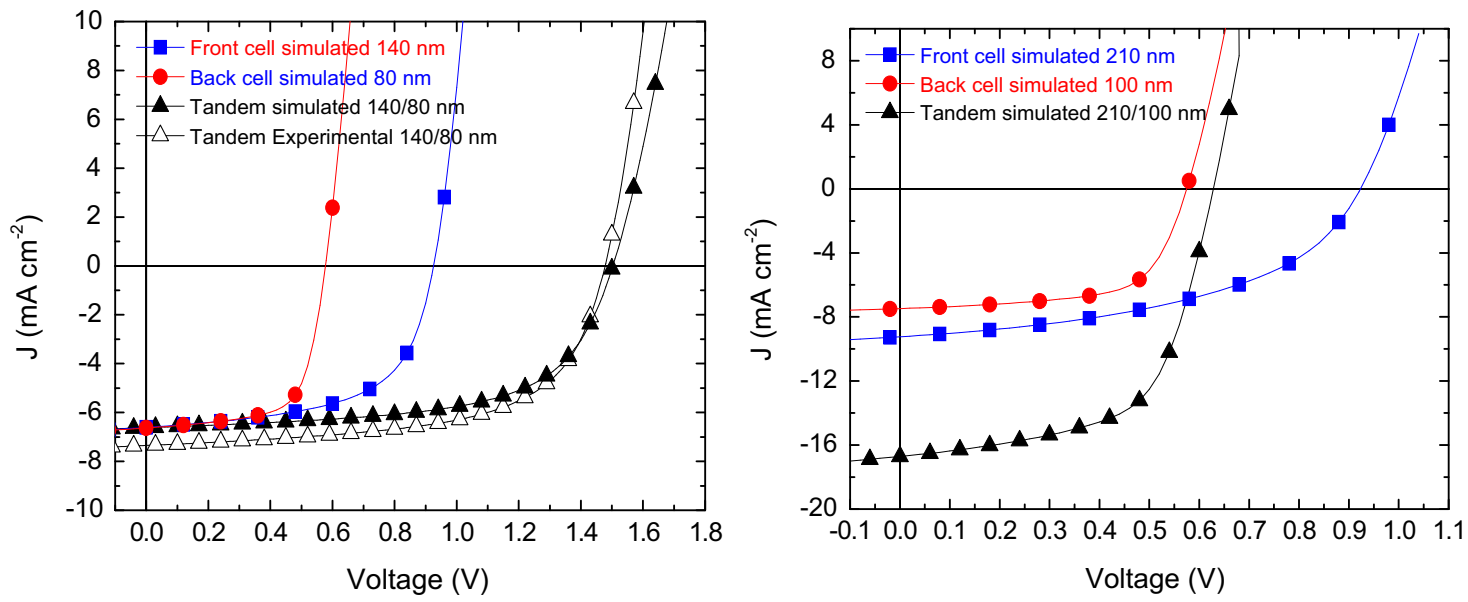

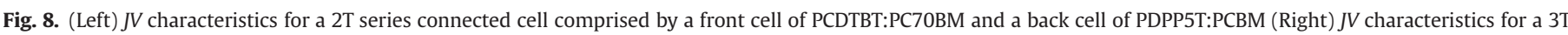
parallel connected cell comprised by a front cell of PCDTBT:PC70BM and a back cell of PDPP5T:PCBM.

Table 4

Figure of merits for the PCDTBT:PC70BM and PDPP5T:PCBM tandem cells shown in Fig. 8 .

\begin{tabular}{llcll}
\hline Device & $\boldsymbol{V}_{\text {oc }}[\mathbf{V}]$ & $\boldsymbol{J}_{\mathbf{s c}}\left[\mathbf{m A ~ \mathbf { ~ c m } ^ { - 2 } ]}\right.$ & FF & PCE [\%] \\
\hline 2T series simulated & 1.50 & 6.93 & 0.65 & 6.8 \\
2T series experimental & 1.48 & 7.41 & 0.61 & 6.7 \\
3T parallel simulated & 0.63 & 16.7 & 0.60 & 6.3 \\
\hline
\end{tabular}

For a $3 \mathrm{~T}$ configuration this would be substrate, screen printed silver, slot die coated PEDOT, ZnO, photoactive layer and PEDOT, inkjet printed silver grid, slot die coated PEDOT, photoactive layer and $\mathrm{ZnO}$, and screen printed silver. It is clear that the experimental devices prepared for this preliminary study do not correspond to the large area module development proposed herein. However, all the interface junctions have been already demonstrated to work and one should not expect unavoidable fundamental 


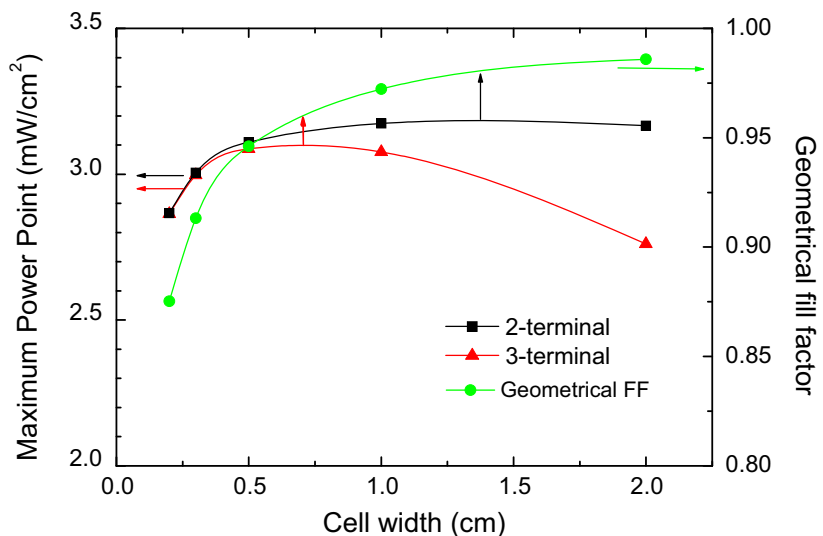

Fig. 9. Maximum power extracted as a function of the cell width (left axis) for $2 \mathrm{~T}$ (black squares) and 3T (red triangles) tandem cells. The geometrical fill factor of modules (green circles) taking into account the cell width restriction is also shown in the right axis. Modules containing 3T cells would have a maximum power extraction for a cell width of $7 \mathrm{~mm}$. This would correspond to a geometrical fill factor of 95\% (red vertical arrow). In contrast, modules with 2T cells would maximize the extracted power at a cell width of $13 \mathrm{~mm}$. This would correspond to a geometrical fill factor of $97 \%$ (black vertical arrow). (For interpretation of the references to color in this figure legend, the reader is referred to the web version of this article.)
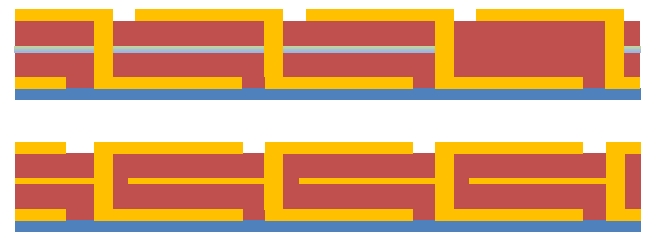

Fig. 10. Schematic designs of interconnection in a $2 \mathrm{~T}$ (top) and $3 \mathrm{~T}$ (bottom) module. The bottom electrode is depicted in blue, the interlayer in the 2T design in gray, active layers in brown and the intermediate electrode for the 3T design and the top electrode for both $2 \mathrm{~T}$ and $3 \mathrm{~T}$ in yellow. (For interpretation of the references to color in this figure legend, the reader is referred to the web version of this article.)

complications when adapting the experimental device process to the large scale manufacturing.

The intermediate electrode in the 3T configuration extracts the sum of the currents photogenerated in both subcells. Since this will be a reasonably large current in comparison to $2 \mathrm{~T}$ tandem devices, the series resistance of the electrode will limit the width of the active area for an efficient charge extraction. For the calculation a $300 \mathrm{~nm}$ thick inkjet printed $\mathrm{Ag}$ grid structure with a pitch of $2.5 \mathrm{~mm}$ was considered as intermediate electrode [20]. Fig. 9 shows the maximum power point of 3T and 2T devices as a function of the cell width. The geometrical fill factor for the corresponding cell width is also shown. Results show that the cell width will be limited to around $5 \mathrm{~mm}$ for optimal performance for 3T tandem cells. In combination with a laser patterning of 200$300 \mu \mathrm{m}$, the geometrical fill factor can be in the range of 95\% (red vertical arrow). 2T devices in contrast, can benefit from geometrical fill factors of $98 \%$ (black vertical arrow) with a similar laser patterning because of a lower current generated in the cell and hence a smaller obstruction by the series resistance.

Regarding R2R module manufacturing, adjacent cells have to be connected in series. The top and bottom electrode of a 3T parallel cell are connected in order to make the cathode of the cell, while the interlayer acts as the anode. The common cathode of one cell can be connected to the intermediate electrode of the following one with a module design whose $\mathrm{R} 2 \mathrm{R}$ manufacturing requires 4 laser steps instead of the 3 needed for a 2T tandem cells based module to open the intermediate electrode for interconnection (Fig. 10).
Furthermore, an excess of energy is needed for the deposition and drying of the additional metallic layer that is used for the interlayer in the 3T design. The more complex module fabrication procedure with an extra laser step plus the excess of electricity needed to process the interlayer do not compensate the potential theoretical efficiency increase that can be expected for 3T in comparison to $2 \mathrm{~T}$ for some material combination.

\section{Conclusion}

In summary, we have presented a comprehensive study and comparison of $2 \mathrm{~T}$ series connected and 3T parallel connected tandem devices for two particular combination of active materials, namely P3HT and PTB7 - fully commercially available, and PCDTBT and PDPP5T - the latter synthesized at the Eindhoven University of Technology. The 2T series connection outperforms the 3T parallel connections. PCE predicted by accurate models show slightly less potential for energy conversion in the 3T design for ideal bandgaps, but superior expected performances for some combinations of donor bandgaps. Experimental devices with the two material combinations chosen for this study always underperformed the results achieved for the $2 \mathrm{~T}$ design. In addition, due to resistive losses at the interlayer and the amount of current that the device has to manage, the cell width must be lower for the 3T, which causes a reduction in the geometrical fill factor of solar modules. Furthermore, the R2R fabrication of such modules requires an additional laser step and an excess of energy to process the interlayer. All together it makes the parallel connection of tandem cells a non-suitable option for the development of organic solar modules.

\section{Acknowledgments}

We thank the European Community's Seventh Framework Programme (FP72007-2013) under Grant no. 287818 of the X10D Project for providing financial support.

\section{References}

[1] Z. He, C. Zhong, S. Su, M. Xu, H. Wu, Y. Cao, Enhanced power-conversion efficiency in polymer solar cells using an inverted device structure, Nat. Photonics 6 (2012) 591-595.

[2] 〈http://www.osadirect.com/news/article/1055/〉.

[3] Y. Liang, Z. Xu, J. Xia, S.-T. Tsai, Y. Wu, G. Li, C. Ray, L. Yu, For the bright future bulk heterojunction polymer solar cells with power conversion efficiency of 7.4\%, Adv. Mater. 22 (2010) E135-E138.

[4] 〈http://www.ise.fraunhofer.de/en/press-and-media/press-releases/presseinfor mationen-2013/world-record-solar-cell-with-44.7-efficiency $>$.

[5] T. Ameri, N. Li, C.J. Brabec, Highly efficient organic tandem solar cells: a follow up review, Energy Environ. Sci. 6 (2013) 2390-2413.

[6] W. Li, A. Furlan, K.H. Hendriks, M.M. Wienk, R.A.J. Janssen, Efficient tandem and triple-juntion polymer solar cells, J. Am. Chem. Soc. 135 (2013) 5529-5532.

[7] J. You, C.-C. Chen, Z. Hong, K. Yoshimura, K. Ohya, R. Xu, S. Ye, J. Gao, G. Li, Y. Yang, 10.2\% Power conversion efficiency polymer tandem solar cells consisting of two identical sub-cells, Adv. Mater. 25 (2013) 3973-3977.

[8] S. Sista, Z. Hong, M.-H. Park, Z. Xu, Y. Yang, High-efficiency polymer tandem solar cells with three-terminal structure, Adv. Mater. 22 (2010) E77-E80.

[9] C. Pacholski, A. Kornowski, H. Weller, Self-assembly of ZnO: from nanodots to hanorods, Angew. Chem. Int. Ed. 41 (2002) 1188-1191.

[10] W.J.E. Beek, M.M. Wienk, M. Kemerink, X. Yang, R.A.J. Janssen, Hybrid zinc oxide conjugated polymer bulk heterojunction solar cells, J. Phys. Chem. B 109 (2005) 9505-9516.

[11] M.S. White, D.C. Olson, S.E. Shaheen, N. Kopidakis, D.S. Ginley, Inverted bulk heterojunction organic photovoltaic device using a solution-derived $\mathrm{ZnO}$ underlayer, Appl. Phys. Lett. 89 (2006) 143517-143523.

[12] G. Dennler, M.C. Scharber, T. Ameri, P. Denk, K. Forberich, C. Waldauf, C. J. Brabec, Design rules for donors in bulk-heterojunction tandem solar cells towards 15\% energy-conversion efficiency, Adv. Mater. 20 (2008) E579-E583.

[13] D. Veldman, S.C.J. Meskers, R.A.J. Janssen, The energy of charge transfer states in electron donor-accpetor blends: insight into the energy losses in organic solar cells, Adv. Funct. Mater. 19 (2009) 1939-1948. 
[14] Y. Kim, S. cook, S.M. Tuladhar, S.A. Choulis, J. Nelson, J.R. Durrant, D.D. C. Bradley, M. Giles, I. McCullogh, C.-S. Ha, M. Ree, A strong regioregularity effect in self-organizing conjugated polymer films and high-efficiency polythiophene:fullerene solar cells, Nat. Mater. 5 (2006) 197-203.

[15] J. Ajuria, I. Etxebarria, W. Cambarau, U. Munecas, R. Tena-Zaera, J.C. Jimeno, R. Pacios, Inverted ITO-free organic solar cells based on $\mathrm{p}$ and $\mathrm{n}$ semiconducting oxides. New designs for integration in tandem cells, top or bottom detecting devices, and photovoltaic windows, Energy Environ. Sci. 4 (2010) 453-458.

[16] J. Gilot, M.M. Wienk, R.A.J. Janssen, Optimizing polymer tandem solar cells, Adv. Mater. 22 (2010) E67-E71.

[17] V.S. Gevaerts, A. Furlan, M.M. Wienk, M. Turbiez, R.A.J. Janssen, Solution processed polymer tandem solar cell using efficient small and wide bandgap polymer:fullerene blends, Adv. Mater. 24 (2012) 2130-2134.
[18] Y. Sun, C.J. Takacs, S.R. Cowan, J.H. Seo, X. Gong, A. Roy, A.J. Heeger, Efficient air-stable bulk heterojunction solar cells using $\mathrm{MoO}_{x}$ as the anode interfacial layer, Adv. Mater. 23 (2011) 2226-2230.

[19] S. Beaupré, M. Leclerc, PCDTBT: en route for low cost plastic solar cells, J Mater. Chem. A 1 (2013) 11097-11105.

[20] Y. Galagan, B. Zimmermann, E.W.C. Coenen, M. Jorgenson, D.M. Tanenbaum, F. C. Krebs, H. Gorter, S. Sabik, L.H. Slooff, S.C. Veenstra, J.M. Kroon, R. Andriessen, Current collecting grids for ito-free solar cells, Adv. Energy Mater. 2 (2011) 103-110. 